\title{
Estructura de la taxocenosis de diatomeas y su relación con variables ambientales en tres cuencas de la provincia de San Luis (Argentina)
}

\author{
Griselda Jorgelina Daruich ${ }^{1}$, María Angélica GiL ${ }^{1}$ y Liliana Elizabeth Moreno²
}

'Área de Biología. Facultad de Química, Bioquímica y Farmacia. UNSL.

2Área de Zoología. Facultad de Química, Bioquímica y Farmacia. UNSL.

E-mail: j.jorgelina@gmail.com

RESUMEN. La composición y distribución de las asociaciones de algas en ecosistemas lóticos son el resultado de condiciones en el medio ambiente y en particular pueden reflejar su estructura y funcionamiento.El objetivo de este trabajo fue estudiar la relación de la riqueza, abundancia y diversidad de las especies de diatomeas con las características ambientales en tres cuencas de la provincia de San Luis. Para ello se seleccionaron 14 sitios de muestreo en tres cuencas: en la cuenca del río Conlara siete sitios, otros tres en la cuenca del río Quinto y finalmente cuatro en la cuenca del Bebedero. En cada sitio de muestreo se registraron parámetros hidráulicos y fisicoquímicos. Se colectaron muestras epilíticas que fueron obtenidas por raspado de pequeñas rocas del fondo. La estructura de la comunidad estuvo caracterizada por el predominio de las diatomeas pennales que se destacaron tanto cualitativa como cuantitativamente. Las variaciones de parámetros fisicoquímicos influyeron en la composición específica de las taxocenosis y permitieron establecer dos grupos de especies: el primero constituido por Nitzschia palea, Melosira varians, Navicula tripunctata y Halamphora veneta que se vincularon a arroyos de mayor número de orden y mayor conductividad. Mientras que Geissleria decussis, Epithemia sorex y Cymbella affinis constituyeron el segundo grupo relacionado con mayor caudal y $\mathrm{pH}$, en tanto que Navicula radiosa y Rophalodia gibba estuvieron asociadas a la altitud y concentración del oxígeno disuelto, respectivamente. Una referencia florística confiable de diatomeas permite hacer diagnósticos fundamentados sobre el estado ecológico de los arroyos, así como para establecer programas de monitoreo en la provincia.

Palabras clave: Diatomeas, estructura, factores fisicoquímicos, ríos serranos.

ABSTRACT. The composition and distribution of algae associations in lotic ecosystems are the result of conditions in the environment and in particular may reflect their structure and functioning. The objective of this work was to study the relationship of the richness, abundance and diversity of the diatom assemblages with the environmental characteristics in three basins of the province of San Luis. Fourteen sampling sites were selected in three basins: seven sites in the Río de Conlara basin, three sites in the Río Quinto basin and finally four sites in the Bebedero basin. In each sampling site, hydraulic and physicochemical parameters were recorded. Epilithic samples were obtained by scraping small rocks from the bottom of the streams. The structure of the assemblages was characterized by pennate diatoms that prevailed qualitatively and quantitatively. The variations of physicochemical parameters influenced the specific composition of the assemblages and allowed to establish two groups of species: one constituted by Nitzschia palea, Melosira varians, Navicula tripunctata and Halamphora veneta that were associated to streams with highest order number and maximum conductivity values. Geiss/eria decussis, Epithemia sorex and Cymbella affinis constituted the second group related to highest discharge and $\mathrm{pH}$ values. Navicula radiosa and Rhopalodia gibba were related to altitude and dissolved oxygen concentration, respectively. A substantiated floristic reference of diatoms allows to make a trustworthy diagnosis on the ecological state of the analyzed streams, as well as to establish monitoring programs for the province.

Keywords: Diatoms, physicochemical factors, streams, structure. 


\section{INTRODUCCIÓN}

El estudio de la estructura y dinámica de las comunidades algales en ríos es complejo debido a la interacción entre variables geomorfológicas, hidrológicas y bióticas que operan en una escala de amplio rango e influyen en la dinámica trófica y ciclo de nutrientes (Galea et al., 2012; Luque y Martínez de Fabricius, 2005; Rout y Gaur, 1994). En particular las taxocenosis de diatomeas son diversas y ocupan un amplio rango de hábitats en estos sistemas. La composición y distribución de las asociaciones de estas algas son el resultado de condiciones en el medio ambiente y en particular pueden reflejar la estructura y funcionamiento de los ecosistemas lóticos (Sabater et al., 1988).

La disponibilidad de los recursos hídricos en los sistemas continentales es limitada y su calidad se ve afectada por distintas causas. El aumento de la población humana y los cambios en el uso de la tierra que ella promueve, constituyen uno de los principales factores que influyen en la biodiversidad a nivel global (Newbold et al., 2015). Para monitorear los cambios ambientales, se opta por las diatomeas respecto a otros grupos porque tienen como ventaja que su colecta es fácil y rápida. La buena conservación de las muestras se debe a la cubierta de sílice -el frústulo- de elevada resistencia y cuyas características morfológicas son la base de la identificación de las especies. Sumado a la amplia distribución del grupo se dispone de una extensa información de las preferencias ecológicas de un considerable número de especies (López Fuerte y Siqueiros Beltrones, 2011). Es conocida la sensibilidad de estos organismos frente a los cambios de las condiciones ambientales por lo cual se los emplea habitualmente como referentes del estado ecológico del ambiente (Martínez de Fabricius, 2000). Los estudios realizados en San Luis, hasta el momento, se enfocaron en la taxocenosis de diatomeas y generaron información de base útil para abordar trabajos de evaluación ambiental (Daruich et al., 2003, 2005; Daruich, 2007). El objetivo de este trabajo fue estudiar la relación entre la riqueza, abundancia y diversidad de la taxocenosis de diatomeas bentónicas con las características ambientales en tres cuencas de la provincia de San Luis con impacto antrópico.

\section{MATERIALES Y MÉTODOS Descripción del área de estudio}

La región estudiada forma parte de las Sierras de San Luis que pertenecen al sistema de sierras pampásicas del centro-oeste de Argentina. La pendiente oriental favorece el desarrollo de una red hidrográfica de relativa importancia donde tienen sus nacientes las tres cuencas del centro de la provincia: del río Conlara, del río Quinto y del Bebedero (Ceci y Cruz Coronado, 1981). La cuenca del río Conlara nace en las sierras de los Comechingones, recorre $180 \mathrm{~km}$, con pendiente hacia el norte en el valle del Conlara, su superficie es de $8800 \mathrm{~km}^{2}$, entre el territorio de la provincia de San Luis y la provincia de Córdoba. La principal actividad es la ganadería de ciclo completo y agricultura de forraje, maíz, soja y papa (Iglesias et al., 2015).

La cuenca del río Quinto con una superficie de $1500 \mathrm{~km}^{2}$ limita al norte con la cuenca del Conlara. Su extensión es de aproximadamente $300 \mathrm{~km}$. El río Quinto nace en la confluencia de los ríos El Trapiche y Grande, que forman el embalse La Florida. Es el recurso hídrico superficial más importante de la provincia. En los últimos años hubo un desarrollo creciente de la agricultura junto a la ganadería. Esta región concentra el $66 \%$ de la agricultura de la provincia (Iglesias et al., 2015). La cuenca del Bebedero está ubicada en la parte central de la provincia, recorre el territorio de la ciudad capital, ocupa un área de $5500 \mathrm{~km}^{2}$, limitada al Este por la divisoria de aguas que la separa de la cuenca superior del río Quinto. Presenta cursos de agua de corto recorrido que se infiltran en los terrenos permeables de piedemonte y escurren de manera subterránea hacia el nivel de base: la laguna del Bebedero (Ceci y Cruz Coronado, 1981). Está formada por las subcuencas de los ríos Potrero y Volcán, ambas reguladas y con distinto grado de actividad antrópica, ya que atraviesan pueblos turísticos en continua expansión. En esta región, los sistemas de producción son predominantemente la ganadería de cría extensiva en monte, además de cultivos agrícolas extensivos con riego y de producción de grano, en especial maíz y soja 


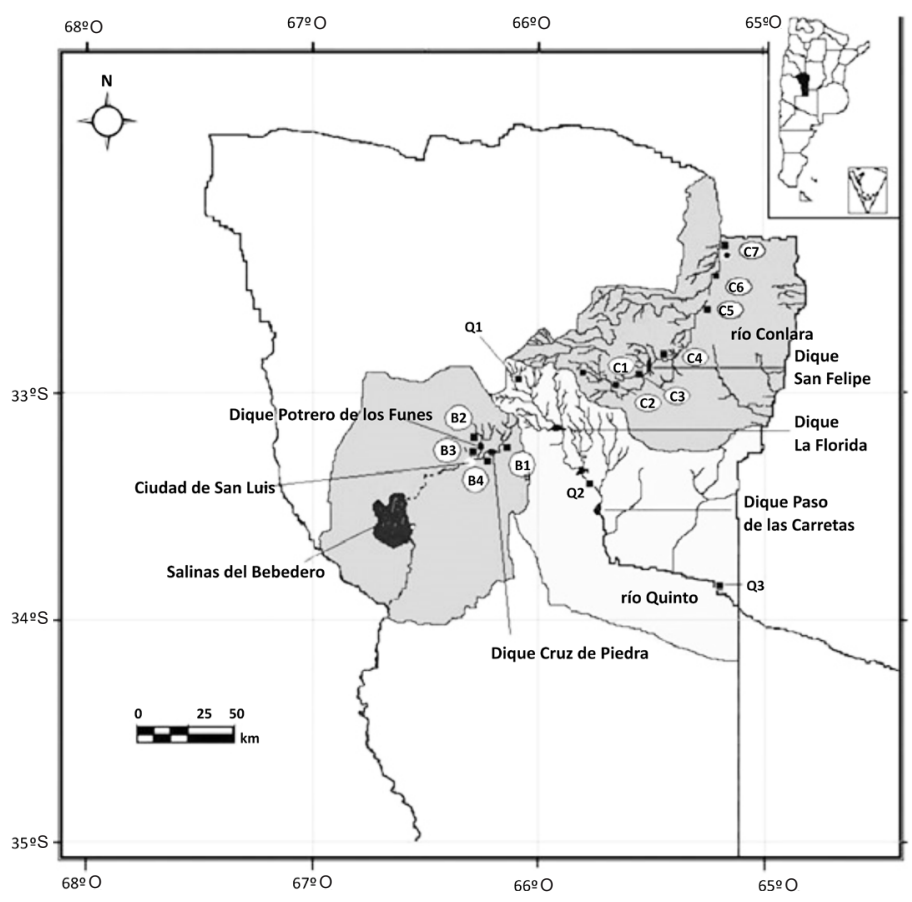

Figura 1. Área de Estudio y localización de los sitios de muestreo.

Figure 1. Study area and location of the sampling stations.

(Iglesias et al., 2015).

Los sistemas fluviales presentan variaciones anuales, con sequías y crecidas directamente relacionadas con el régimen de precipitaciones. Las lluvias son de tipo estival y tienen lugar casi en su totalidad en el período octubre-marzo, con medias anuales entre 500 y $650 \mathrm{~mm}$. El clima es templado seco; la zona en estudio posee características de semiaridez, por ello la conservación del recurso resulta de gran importancia tanto para los asentamientos humanos como para la flora y la fauna.

Para este estudio se seleccionaron 14 sitios de muestreo en tres cuencas de la provincia de San Luis (Figura 1): siete en la cuenca del río Conlara: C1, C2, C3, C4, C5, C6, C7; tres en la Cuenca del río Quinto: Q1, Q2 y Q3 y cuatro en la cuenca del Bebedero (dos en el río Potrero: B1y B4, y dos en el río Volcán: B2 y B3). Los centros urbanos de mayor densidad poblacional próximos a los sitios de muestreo son: Concarán (5.119 habitantes) y Santa Rosa del Conlara (10.461 habitantes) en los sitios C5 y C7 respectivamente; Villa Mercedes, de
111.321 habitantes en el sitio Q3; Potrero de los Funes y ciudad de San Luis, de 1.698 y 169.947 habitantes, respectivamente en los sitios B2 y B4 (INDEC, 2010).

En cada sitio se registraron parámetros hidráulicos del cauce: ancho y velocidad de corriente y se calculó el caudal. Los parámetros fisicoquímicos se midieron in situ con sensores portátiles: CONSORT modelo C532, el pH (exactitud $\pm 0,01 \mathrm{pH}$ ), la conductividad (exactitud $\pm 1 \mu \mathrm{S} . \mathrm{cm}^{-1}$ ) y la temperatura (exactitud $\pm 0,5^{\circ} \mathrm{C}$ ) y el $\%$ de saturación de oxígeno (exactitud $\pm 0,01$ mg. $\mathrm{L}^{-1}$ ) con CONSORT modelo Z921.

\section{Muestreo de diatomeas}

Las campañas de muestreo se llevaron a cabo en temporada de bajo y de alto caudal (febrero-marzo y agosto-septiembre de 2011, respectivamente). Las muestras se recolectaron al azar tomando pequeñas rocas del fondo y si el lecho se presentaba limoso arenoso, se procedió a extraer una alícuota del sedimento con un aspirador manual con $2 \mathrm{~cm}^{2}$ de superficie 
(Venrick, 1995).

En el laboratorio, cada una de las muestras fueron tratadas con agua oxigenada de 100 vol. a $80^{\circ} \mathrm{C}$ para limpieza de las tecas silíceas (Battarbee, 1986). Los recuentos de las diatomeas se efectuaron con microscopio óptico con aumento de 400X mientras que la identificación se realizó con una magnificación de 1000X usando aceite de inmersión (Gómez et al., 2009). Se contaron 200 valvas en cada muestra (Bate \& Newall, 2002). Se empleó Naphrax ${ }^{\circledR}$ como medio de montaje en los preparados diatomológicos permanentes según las técnicas de Hasle \& Fryxel (1970). Para la identificación de especies se utilizaron trabajos de Krammer \& Lange-Bertalot (1986, 1988, 1991a, 1991b) y varios de flora diatomológica regional (Martínez de Fabricius y Corigliano, 1989; Martínez de Fabricius, 1996; Luque et al., 1997; Martínez de Fabricius et al., 2005; Daruich, 2007). Además, se consultó la base de datos registrada en el sitio web Algabase (Guiry \& Guiry 2016).

\section{Análisis de datos}

Para cada sitio de muestreo se estimaron la abundancia relativa de cada especie, la riqueza específica y el índice de diversidad de ShannonWiener $\left(\mathrm{H}^{\prime}\right)$.

Se determinó una longitud de gradiente alta, y bajo el supuesto de simetría, se optó por un CCA (Análisis de Correspondencia Canónica), construyéndose una matriz que contiene los descriptores ambientales y la abundancia de especies en cada uno de los sitios de muestreo. El análisis se realizó con PAST v.2.0 (Hammer et al., 2001).Para reducir el efecto de la variación, la abundancia de los taxones se transformó con la raíz cuadrada, mientras que todos los datos ambientales, excepto el $\mathrm{pH}$ y el número de orden del río, se transformaron logarítmicamente para reducir la distribución sesgada (Leira \& Sabater, 2005).

\section{RESULTADOS}

La información referida a las variables ambientales, fisicoquímicas e hidrológicas registradas en ambos periodos de muestreos para cada sitio no mostró diferencias significativas por lo que los valores fueron promediados (Tabla 1).

Las diatomeas estuvieron representadas por un total de 66 especies agrupadas en 34 géneros de los cuales los más representativos fueron Navicula, Nitzschia y Pinnularia. A cada especie se les asignó el acrónimo correspondiente. Algunas especies se presentaron de manera abundante como Diatoma vulgaris Bory, Cymbella affinis Kützing y Ulnaria ulna (Nitzsch) Compère (UULN) mientras que otras como Halamphora veneta (Kützing) Levkovy Pinnularia maior (Kützing) Rabenhorst fueron raras.

El análisis de la diversidad evidenció similitudes en las nacientes de las tres cuencas y en las desembocaduras, siendo los sitios menos diversos C7 $\left(H^{\prime}=1,64\right)$ y Q3 $\left(H^{\prime}=1,70\right)$ (Figura 2$)$. Los resultados revelaron que la riqueza de especies fue mayor en las nacientes, es decir en los tramos de orden inferior, y disminuyó hacia los tramos de orden superior, encontrándose la menor riqueza específica en los sitios C7 y Q3 (Figura 3).

El análisis de correspondencia canónico mostró un $54,43 \%$ de la variación explicada por la conductividad y el número de orden del arroyo y el $34,98 \%$ de la variación se explica por el $\mathrm{pH}$ y el caudal (Figura 4). La conductividad y el número de orden del arroyo agruparon a las especies Navicula tripunctata (O.F.Müller) Bory (NTPT), Nitzschia palea (Kützing) W. Smith (NPAL) y Melosira varians C.Agardh (MVAR) mientras que el $\mathrm{pH}$ y el caudal a Epithemia sorex Kützing (ESOR), Cymbella affinis (CAFF) y Diatoma vulgaris (DVUL). Navicula radiosa Kützing (NRAD) y Rhopalodia gibba (Ehrenberg) O. Müller (RGIB) estuvieron parcialmente relacionadas con la altitud y el oxígeno disuelto, respectivamente. La relación de las especies con las variables ambientales permitió establecer dos grupos de especies: a) especies de arroyos con mayor número de orden y mayores valores de conductividad: N. palea, M. varians, N. tripunctata y Halamphora veneta (HVEN) y b) especies asociadas a mayor caudal y $\mathrm{pH}$ como Geissleria decussis (Østrup) Lange-Bertalot \& Metzeltin (GDEC), E. sorex y C. affinis.

\section{DISCUSIÓN}

La estructura de la taxocenosis de diatomeas 
Tabla 1. Valores medios ( \pm desviación estándar) de variables hidrológicas y fisicoquímicas de los sitios analizados en las tres cuencas de la provincia de San Luis.

\begin{tabular}{|c|c|c|c|c|c|c|c|c|}
\hline Sitio & Ubicación & $\begin{array}{l}\text { Número } \\
\text { orden }\end{array}$ & $\begin{array}{l}\text { Caudal } \\
\left(\mathrm{m}^{3} \mathrm{~s}^{-1}\right)\end{array}$ & $\begin{array}{l}\text { Altitud } \\
\text { (msnm) }\end{array}$ & $\begin{array}{c}\text { Temperatura } \\
\left(\mathrm{C}^{\circ}\right)\end{array}$ & $\mathrm{pH}$ & $\begin{array}{c}\text { Oxígeno } \\
\text { disuelto } \\
\left(\mathrm{mg} \mathrm{l}^{-1}\right)\end{array}$ & $\begin{array}{l}\text { Conductividad } \\
\left(\mu \mathrm{Sm}^{-1}\right)\end{array}$ \\
\hline \multirow[t]{2}{*}{ C1 (Arroyo Luluara) } & $32^{\circ} 46^{\prime} \mathrm{S}$ & $1^{\circ}$ & $0,82 \pm 0,5$ & 1160 & $15,25 \pm 6,40$ & $8,07 \pm 0,7$ & $10,98 \pm 0,4$ & $267,2 \pm 21,5$ \\
\hline & $65^{\circ} 50^{\prime} \mathrm{O}$ & & & & & & & \\
\hline \multirow[t]{2}{*}{ C2 (Paso Grande) } & $32^{\circ} 53^{\prime} \mathrm{S}$ & $3^{\circ}$ & $1,17 \pm 0,9$ & 957 & $17,12 \pm 4,97$ & $7,76 \pm 0,5$ & $10,67 \pm 0,4$ & $308,5 \pm 25,2$ \\
\hline & $65^{\circ} 38^{\prime} \mathrm{O}$ & & & & & & & \\
\hline \multirow{2}{*}{$\begin{array}{l}\text { C3 (anterior Dique San } \\
\text { Felipe) }\end{array}$} & $32^{\circ} 54^{\prime} \mathrm{S}$ & $4^{\circ}$ & $1,21 \pm 0,7$ & 863 & $20,87 \pm 6,86$ & $7,48 \pm 0,8$ & $9,27 \pm 1,0$ & $346,0 \pm 41,9$ \\
\hline & $65^{\circ} 31^{\prime} \mathrm{O}$ & & & & & & & \\
\hline \multirow{2}{*}{$\begin{array}{l}\text { C4 (posterior Dique San } \\
\text { Felipe) }\end{array}$} & $32^{\circ} 47^{\prime} \mathrm{s}$ & $4^{\circ}$ & $0,79 \pm 0,3$ & 798 & $15,90 \pm 7,52$ & $7,5 \pm 0,6$ & $9,40 \pm 1,6$ & $421,0 \pm 62,6$ \\
\hline & $65^{\circ} 25^{\prime} \mathrm{O}$ & & & & & & & \\
\hline \multirow[t]{2}{*}{ C5 (Concarán) } & $32^{\circ} 33^{\prime} \mathrm{S}$ & $4^{\circ}$ & $0,22 \pm 0,1$ & 662 & $16,95 \pm 7,00$ & $7,23 \pm 0,3$ & $8,94 \pm 1,2$ & $1984,7 \pm 343,6$ \\
\hline & $65^{\circ} 15^{\prime} \mathrm{O}$ & & & & & & & \\
\hline C6 (Ojo de río) & $\begin{array}{l}32^{\circ} 26^{\prime} \mathrm{S} \\
65^{\circ} 14^{\prime} \mathrm{O}\end{array}$ & $4^{\circ}$ & $0,38 \pm 0,3$ & 613 & $19,70 \pm 5,61$ & $6,94 \pm 0,8$ & $9,42 \pm 1,8$ & $2372,5 \pm 342,4$ \\
\hline $\begin{array}{l}\text { C7 (Santa Rosa del } \\
\text { Conlara) }\end{array}$ & $\begin{array}{l}32^{\circ} 20^{\prime} \mathrm{S} \\
65^{\circ} 13^{\prime} \mathrm{O}\end{array}$ & $4^{\circ}$ & $0,66 \pm 0,6$ & 575 & $14,80 \pm 6,01$ & $6,63 \pm 0,6$ & $8,01 \pm 1,7$ & $1864,2 \pm 96,5$ \\
\hline B1 (La Estrechura) & $\begin{array}{l}33^{\circ} 14^{\prime} \mathrm{S} \\
66^{\circ} 12^{\prime} \mathrm{O}\end{array}$ & $2^{\circ}$ & $0,23 \pm 0,1$ & 992 & $17,4 \pm 2,2$ & $8,12 \pm 0,4$ & $12,73 \pm 2,4$ & $447,2 \pm 332,1$ \\
\hline B2 (Cuchi Corral) & $\begin{array}{l}33^{\circ} 17^{\prime} \mathrm{S} \\
66^{\circ} 15^{\prime} \mathrm{O}\end{array}$ & $3^{\circ}$ & $0,14 \pm 0,15$ & 799 & $16,3 \pm 4,9$ & $8,07 \pm 0,1$ & $8,50 \pm 2,3$ & $131,7 \pm 31,6$ \\
\hline B3 (Los Molles) & $\begin{array}{l}33^{\circ} 12^{\prime} \mathrm{S} \\
66^{\circ} 14^{\prime} \mathrm{O}\end{array}$ & $2^{\circ}$ & $0,19 \pm 0,1$ & 1000 & $15,2 \pm 4,1$ & $7,62 \pm 0,4$ & $6,88 \pm 1,0$ & $380,7 \pm 88,9$ \\
\hline \multirow[t]{2}{*}{ B4 (Las Chacras) } & $33^{\circ} 14^{\prime} \mathrm{S}$ & $3^{\circ}$ & $0,18 \pm 0,07$ & 903 & $17,3 \pm 2,12$ & $7,32 \pm 0,2$ & $7,30 \pm 0,99$ & $972,5 \pm 219,9$ \\
\hline & $66^{\circ} 14^{\prime} \mathrm{O}$ & & & & & & & \\
\hline \multirow[t]{2}{*}{ Q1 La Carolina } & $32^{\circ} 48^{\prime} \mathrm{S}$ & $4^{\circ}$ & $0,52 \pm 0,2$ & 1700 & $12,75 \pm 4,03$ & $8,15 \pm 0,4$ & $8,92 \pm 0,30$ & $188,5 \pm 21,6$ \\
\hline & $66^{\circ} 58^{\prime} \mathrm{O}$ & & & & & & & \\
\hline Q2 Saladillo & $\begin{array}{l}33^{\circ} 14^{\prime} \mathrm{S} \\
65^{\circ} 54^{\prime} \mathrm{O}\end{array}$ & $4^{\circ}$ & $1,37 \pm 0,6$ & 613 & $13,00 \pm 3,65$ & $8,19 \pm 0,5$ & $8,55 \pm 0,50$ & $196,0 \pm 20,2$ \\
\hline \multirow[t]{2}{*}{ Q3 Villa Sales } & $33^{\circ} 50^{\prime} \mathrm{S}$ & $6^{\circ}$ & $0,45 \pm 0,2$ & 429 & $11,25 \pm 2,22$ & $7,62 \pm 0,4$ & $5,75 \pm 0,49$ & $3394,2 \pm 380,8$ \\
\hline & $65^{\circ} 14^{\prime} \mathrm{O}$ & & & & & & & \\
\hline
\end{tabular}

estuvo caracterizada por las diatomeas pennales que se destacaron tanto cualitativamente como cuantitativamente coincidiendo con lo registrado en otros ríos serranos de San Luis (Daruich, 2007) y Córdoba (Luque y Martínez de Fabricius, 2000, 2003, 2005). Si bien la diatomea céntrica $M$. varians es conocida por su hábito bentónico, solo resultó dominante en Los Molles (sitio B3). Este punto de muestreo forma parte de la subcuenca del río Potrero regulada por el embalse Potrero de los Funes que presenta señales de eutrofización (Almeida et al., 2007) debido al creciente desarrollo turístico de la zona. Melosira varians ha sido caracterizada como colonizadora primaria estableciendo importantes colonias en un entorno donde existen condiciones de bajo caudal y con acumulación de materia orgánica (en Argentina: Seeligmann et al., 2001 y Mengo, 2017, en Colombia: Martínez, 2003).

La diversidad específica no es estrictamente una medida de impacto en los ríos, pero es una herramienta valiosa para medir la estabilidad de un ecosistema y cuantificar los efectos que las 


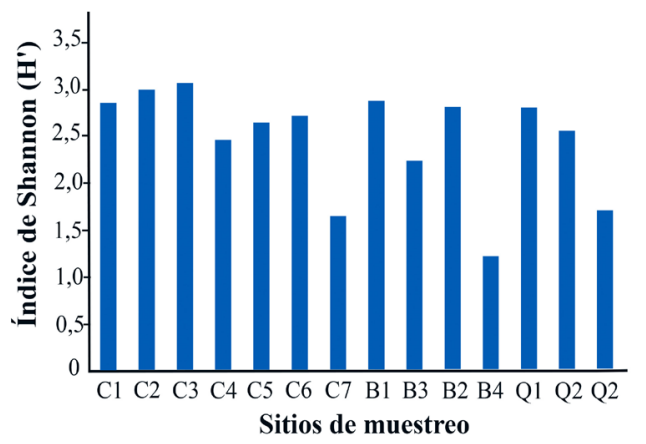

Figura 2. Valores promedio del Índice de diversidad $\left(\mathrm{H}^{\prime}\right)$ de las diatomeas registradas en cada sitio de muestreo de las tres cuencas de la provincia de San Luis.

Figure 2. Diversity index $\left(\mathrm{H}^{\prime}\right)$ mean values of the diatoms recorded at each sampling station from the three basins of San Luis province.

perturbaciones podrían tener en los sistemas naturales. En coincidencia con Mora-Navarro et al. (2004) se registraron valores mayores de diversidad y riqueza en los sitios más alejados de asentamientos urbanos donde las condiciones naturales posibilitan la presencia de mayor cantidad/diversidad de microhábitats que proporcionan protección a los efectos de deriva. La diversidad presentó los valores menores en

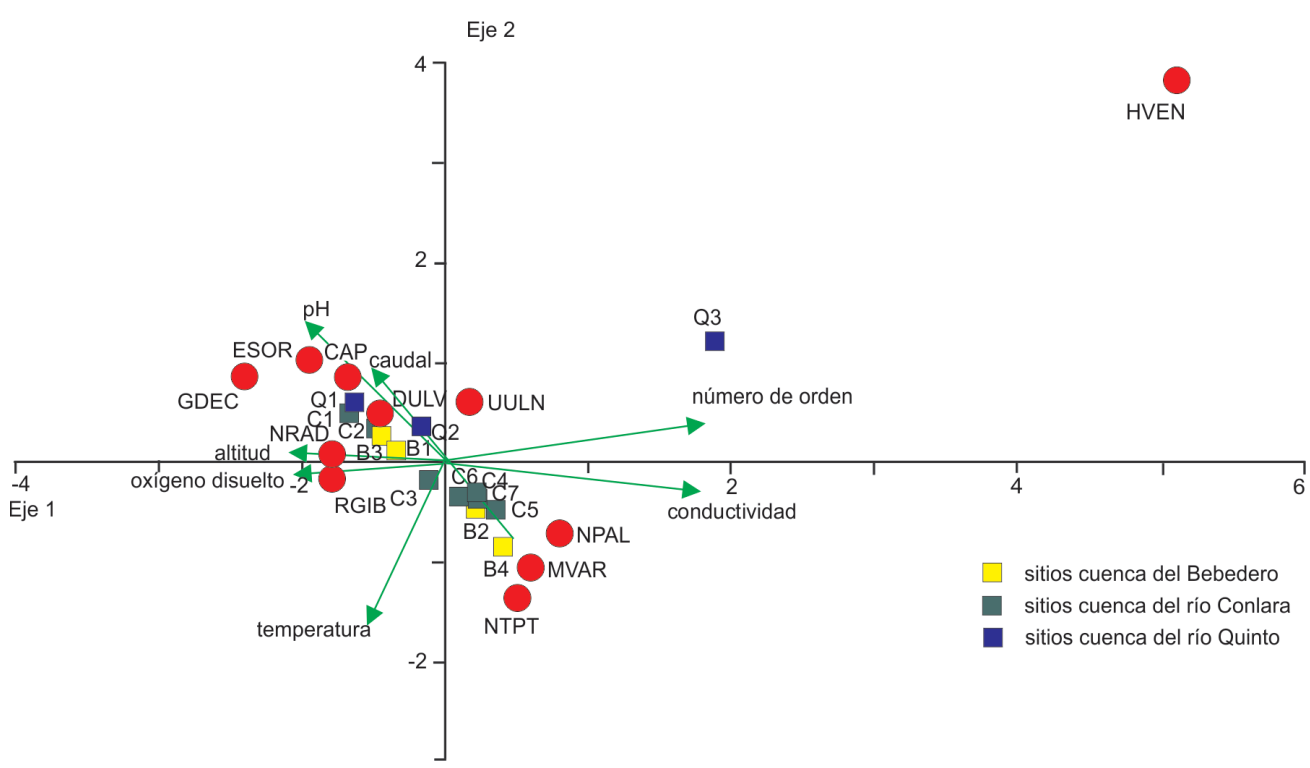

Figura 4. Ordenamiento obtenido con el Análisis de Correspondencia Canónica (ACC) que muestra la distribución de las diatomeas en función de variables ambientales de los arroyos estudiados en las tres cuencas de San Luis.

Figure 4. Results of the canonical correspondence analysis (CCA) showing the diatom distribution related to environmental variables at the selected streams from the three basins of San Luis province. 
evidenciaron un grave deterioro de la calidad biológica estimada con el Índice Biótico del Carcarañá (I.B.C.) y con el ensamble de peces empleado por Garelis y Bistoni (2010), en ambos casos atribuido al vertido de efluentes cloacales e industriales.

Las variaciones de los factores fisicoquímicos influyeron en la composición específica de la taxocenosis de diatomeas y permitieron establecer dos grupos de especies. El primero conformado por N. palea, M. varians, N. tripunctata y $H$. veneta se asoció a arroyos de mayor número de orden y mayor conductividad, sugiriendo que estas especies están adaptadas por su metabolismo a estas condiciones o bien tienen la capacidad de tolerar considerables niveles de conductividad. Este factor puede además estar afectado por contaminación, movimiento del agua y la morfología e hidrología del cuerpo del agua que también determinan la estructura de la taxocenosis (Huszar et al., 1998). Nitzschia palea ha sido relacionada con ambientes polisapróbicos o hipereutrofizados y capaz de vivir en ambientes someros sometidos a la desecación (Lobo et al., 1996; Sabater et al., 1988; Salomoni et al., 2006; Sosa et al., 2011). En Argentina, Hassan (2004) y Gómez y Licursi (2001) señalaron que $H$. veneta y $N$. tripunctata se relacionan a ambientes con alto contenido de materia orgánica. El segundo grupo constituido por G. decussis, E. sorex y C. affinis se asoció a mayor caudal y $\mathrm{pH}$. La dominancia de $C$. affinis en aguas con elevado $\mathrm{pH}$ se ha observado en otros estudios realizados para la provincia de San Luis (Daruich, 2007). El aumento de caudal favoreció la presencia de E. sorex, pudiendo adherirse también al sedimento y no sólo a la vegetación presente (Tiffany, 2011). Ulnaria ulna, a pesar de ser una especie reófila, tuvo también una destacada presencia en sistemas con baja velocidad de corriente, por lo que su registro responde a un amplio intervalo de condiciones ambientales (Sala, 1996; Salomoni et al., 2006; Sosa et al., 2011). R. gibba y N. radiosa se destacaron en los sitios de mayor altitud, alejados de los asentamientos urbanos y de difícil acceso. A $R$. gibba es común encontrarla adherida a macrófitas sumergidas en aguas claras donde hay una buena disponibilidad de oxígeno (Cano, 2008), aunque también puede ser bentónica (Tiffany, 2011). Cabe destacar que el género Navicula es extremadamente común y que difícilmente pueda tomarse una muestra de epipelon sin encontrarlo (Round et al., 1990). Coincidentemente con esto, en los ambientes de altura de la Argentina, ha sido el género más frecuente y representado por el mayor número de especies, con una amplia distribución geográfica incluyendo a $N$. radiosa (Seeligmann y Maidana, 2013).

Puede concluirse que el conocimiento de las taxocenosis de diatomeas registradas en los sistemas lóticos analizados provee información acerca de características relevantes de diferentes tramos de cada una de las cuencas estudiadas y permite sentar bases para monitoreos biológicos en la provincia de San Luis.

\section{AGRADECIMIENTOS}

El presente trabajo fue financiado por el Proyecto 2-3514 "Biota Asociada a los Humedales del centro oeste de Argentina" de la Secretaría de Ciencia y Técnica de la Facultad de Química, Bioquímica y Farmacia de la Universidad Nacional de San Luis. Las autoras de este trabajo agradecen a los revisores por sus oportunas sugerencias y al Profesor Martín Gonzalo Zapico por su asesoramiento estadístico. Asimismo, desean reconocer el aporte de las Dras. Susana Tripole y Patricia Garelis (in memorian), por sentar las bases para la realización del presente trabajo.

\section{REFERENCIAS}

Almeida, C., Quintar, S. González, P. \& Mallea, M. (2007). Influence of urbanization and tourist activities on the water quality of the Potrero de los Funes River (San Luis - Argentina). Environmental Monitoring and Assessment, 133: 459-465.

Bate, N. \& Newall, P. (2002). Techniques for the use of diatoms in water quality assessment: How many valves? In: John, J. (Ed.). Proceedings of the 15th International Diatom Symposium (pp. 153-160). Liechtenstein: A.R.G. Gantner Verlag. 
Battarbee, R. W. (1986). Diatom analysis. In: Berglund, B. E. (Ed.). Handbook of Holocene Palaeoecology and palaeohydrology (pp. 527570). Chichester: John Wiley and Sons.

Cano, M. G. (2008). Fitoperifiton de un lago somero y su relación con los estados de biequilibrio. Tesis doctoral $N^{\circ}$ 1033. Universidad Nacional de La Plata.

Ceci, J. H. \& Cruz Coronado, M. D. (1981). Recursos Hídricos Subterráneos. En: Yrigoyen, M. (Ed.). Geología y Recursos Minerales de la Provincia de San Luis. Relatorio VIII Congreso Geológico Argentino: 301-322.

Daruich, J. (2007). Composición y Distribución de las Bacillariophyceae de la cuenca del río Nogolí, San Luis, Argentina. Tesis Doctoral. Universidad Nacional de San Luis.

Daruich, J., Nievas, C. M. \& Martínez de Fabricius, A. L. (2003). Variación en la composición de Diatomeas de la Cuenca del río Nogolí (San Luis, Argentina). Boletín de la Sociedad Argentina de Botánica, 38 (Supl.): 150.

Daruich, J., Martínez de Fabricius, A. L. \& Ulacco, J.H. (2005). Epilithic algae from Nogoli River (San Luis, Province). Biocell, 29: 360.

Galea, M., J., Álvarez, S. B. Bazán, G. I. \& Martínez de Fabricius, A. L. (2012). Monitoreo inicial de la ficoflora en Villa Vieja, Río Colorado (Dpto. Puelén, La Pampa, Argentina). Biología Acuática, 27: 135-140.

Garelis, P. A. \& Bistoni, M. de los A. (2010). Ictiofauna de la cuenca endorreica del río Quinto (San Luis, Argentina). Natura Neotropicalis, 41(1-2): 19-30.

Gómez, N. \& Licursi, M. (2001). The Pampean Diatom Index (IDP) for assessment of rivers and streams in Argentina. Aquatic Ecology, 35: 173-181.

Gómez, N., Donato, C. J., Giorgi, A., Guasch, H., Matero, P. y Sabater, S. (2009). La biota de los ríos: los microorganismos autótrofos. En: Elosegui, A. y Sabater, S. (Eds.). Conceptos y técnicas de ecología fluvial (pp. 219-238). Bilbao: Fundación BBVA.
Guiry, M. D. \& Guiry, G. M. (2016). Algae Base. World-wide electronic publication, National University of Ireland, Galway. Recuperado en: http://www.algaebase.org. (5-09-2019).

Hammer, O., Harper, D. A. T. \& Ryan, P. D. (2001). PAST: Paleontological Statistic software package for education and data analysis. Paleontologia Eletronica 4 (1): 1-9.

Hasle, G. R. \& Fryxel, A. (1970). Diatoms; cleaning and mounting for light and electron microscopy. Transactions of the American Microscopical Society, 89: 469-474.

Hassan, G. S., Espinosa, M. A. e Isla, F. I. (2004). Distribución de diatomeas en el estuario del río Quequén Grande (Buenos Aires, Argentina). Actas // Reunión Binacional de Ecología (Argentina Chile) Ecología en tiempos de cambios, Mendoza: 422.

Huszar, V. L. M., Silva, L. H. S., Domingos, P., Marinho, M. \& Melo, S. (1998). Phytoplankton species composition is more sensitive than OECD criteria to the trophic status of three Brazilian tropical lakes. Hydrobiologia, 369/370: 59-71.

Iglesias, D., Manazza, F., Belgrano Rawson, A. y Guerri, E. (2015). Márgenes brutos de los principales productos agropecuarios de la provincia de San Luis. Boletín Económico Redes de Economía Agropecuaria de La Pampa y San Luis. Villa Mercedes: Instituto Nacional de Tecnología Agropecuaria.

INDEC. (2010). Censo Nacional de Población, Hogares y Vivienda. Recuperado en: http://www. indec. gob.ar. (fecha de acceso: 20-9-2019).

Krammer, K. \& Lange-Bertalot, H. (1986). Bacillariophyceae. 1. Teil: Naviculaceae. In: Ettl, H., Gerloff, J., Heynig, H. \& Mollenhauer, D. (Eds). Süsswasser flora von Mitteleuropa, 2/1 (pp 1-876). Stuttgart, New York: Gustav Fischer Verlag.

Krammer, K. \& Lange-Bertalot, H. (1988). Bacillariophyceae. 2. Teil: Bacillariaceae, Epithemiaceae, Surirellaceae. In: Ettl, H., Gerloff, J., Heynig, H. \& Mollenhauer, D. (Eds). Süsswasserflora von Mitteleuropa, 2/2 (pp. 1-596). Jena: VEB Gustav Fischer Verlag. 
Krammer, K. \& Lange-Bertalot, H. (1991a). Bacillariophyceae. 3. Teil: Centrales, Fragilariaceae, Eunotiaceae. In: Ettl, H., Gerloff, J., Heynig, H. \& Mollenhauer, D. (Eds). Süsswasserflora von Mitteleuropa, 2/3 (pp 1-576). Stuttgart, Jena: Gustav Fischer Verlag.

Krammer, K. \& Lange-Bertalot, H. (1991b). Bacillariophyceae. 4. Teil: Achnanthaceae, Kritische Ergänzungen zu Navicula (Lineolatae) und Gomphonema, Gesamtliteraturverzeichnis Teil 1-4. In: Ettl, H., Gärtner, G., Gerloff, J., Heynig, H. \& Mollenhauer, D. (Eds). Süsswasserflora von Mitteleuropa, 2/4 (pp. 1-437). Stuttgart, Jena: Gustav Fischer Verlag.

Leira, L. \& Sabater, S. (2005). Diatom assemblages distribution in Catalan rivers NE Spain in relation to chemical and physiographical factors. Water Research, 39: 73-82.

Lobo, E. A., Callegaro, V. L. M., Oliveira, M. A., Salomoni, S. E., Schuler, S. \& Asai, K. (1996). Pollution Tolerant Diatoms from Lotic Systems in the Jacui Basin, Rio Grande do Sul, Brasil. Iheringia Série Botânica, 47: 45-72.

López Fuerte, F. O. \& Siqueiros Beltrones, D.A. (2011). Diatomeas como indicadores de la calidad ecológica de los oasis de Baja California Sur, México. CONABIO. Biodiversitas, 99: 8-1.

Luque, M. E., Gari, E. N. y Martínez de Fabricius, A. L. (1997). Fitoplancton y fitobentos de la cuenca superior del río Chocancharava (ex Cuarto) (Córdoba, Argentina). Revista UNRC, 17(1): 49-67.

Luque, M. E. y Martínez de Fabricius, A. L. (2000). Ficoflora fitoplanctónica y epilítica del río Piedra Blanca (Córdoba, Argentina). Boletín de la Sociedad Argentina de Botánica, 35(1-2): 21-32.

Luque, M. E. y Martínez de Fabricius, A. L. (2003). Distribución temporal del fitoplancton y epiliton en el río Piedra Blanca (Córdoba, Argentina). Limnetica, 22 (3-4): 19-34.

Luque, M. E. y Martínez de Fabricius, A. L. (2005). Algas fitoplanctónicas del Río Piedra Blanca (Córdoba, Argentina) y su relación con los factores ambientales. Lilloa, 42(1-2): 69-79.
Martínez, L. F. (2003). Efectos del caudal sobre la colonización de algas en un río de alta montaña tropical (Boyacá, Colombia). Caldasia, 25(2): 337-354.

Martínez de Fabricius, A. L. (1996). Bacillariophyceae del río Cuarto. Provincia de Córdoba, Argentina. Tesis Doctoral $N^{\circ} 673$, Universidad Nacional de La Plata.

Martínez de Fabricius, A. L. (2000). Las algas, indicadores de la calidad del agua. Interciencia, UNRC, Río Cuarto, 4(4): 1-4.

Martínez de Fabricius, A. L. y Corigliano, M. C. (1989). Composición y distribución de comunidades algales en el río Ctalamochita (Córdoba, Argentina). Revista UNRC, 9:5-13.

Martínez de Fabricius, A. L., Luque, M. E. \& Boccolini, M. (2005). Diatomeas planctónicas de cursos de agua: Cuenca del Río Piedra Blanca (Córdoba, Argentina). Boletín Sociedad Argentina Botánica, 40 (3-4): 183-198.

Mengo, L. (2017). Composición taxonómica y riqueza de diatomeasen el Río Suquía, Córdoba (Argentina). Revista Facultad de Ciencias Exactas, Físicas y Naturales, 4:55-62.

Mora-Navarro M. R., Vázquez-García J. A. y Vargas-Rodríguez Y. L. (2004). Ordenación de comunidades de fitoplancton en el lago de Chapala, Jalisco-Michoacán, México. Hidrobiológica, 14 (2): 91-103.

Newbold, T., Hudson, L. N., Hill, S. L. L., Contu, S., Lysenko, I., Senior, R. A., Börger, L., Dominic Bennett, D. J., Choimes, A., Collen, B., Day, J., De Palma, A., Díaz, S. \& Echeverria-Londoño, S. (2015). Global effects of land use on local terrestrial biodiversity. Nature, 520: 45-50.

Round, F. E., M. Crawford \& Mann, D. G. (1990). The diatoms. Biology and morphology of the genera. Cambridge University Press, Cambridge.

Rout. J. \& Gaur, J.P. (1994). Composition and dynamics of epilithic algae in a forest stream at Shillong (India). Hydrobiologia, 291: 61-74. 
Sabater, S., Sabater, F. \& Armengol, J. (1988). Comunidades de diatomeas en localidades altamente contaminadas del río Ter (Cataluña, NE España). Limnetica, 3:103-109.

Sala, S. E. (1996). Flora diatomológica del embalse Paso de las Piedras (Argentina) I: Fam. Diatomaceae, Fam. Achnantaceae y Fam. Eunotiaceae (O. Pennales). Darwiniana, 34(1-4): 251-266.

Salomoni, S. E., Rocha, O., Callegaro, V. L. \& Lobo, E. A. (2006). Epilithic diatoms as indicators of water quality in the Gravatí River, Rio Grande do Sul, Brazil. Hydrobiologia, 559: 233-246.

Seeligmann, C., Tracanna. B. C., Martínez de Marco, S. y Isasmendi, S. (2001). Algas fitoplanctónicas en la evaluación de la calidad del agua de sistemas lóticos en el noroeste argentino. Limnetica, 20(1): 123-133.
Seeligmann, C. y Maidana, N. 2013. ¿Existen especies de Navicula (Bacillariophyta) exclusivas de ambientes de alta montaña en Argentina? Boletín de la Sociedad Argentina de Botánica. 48 (3-4): 421-433. 2013

Tiffany, M. A. (2011). Epizoic and epiphytic diatoms. In: Seckbach, J. \& Kociolek, P. (Eds.). The Diatom World (pp. 195-209). Dordrecht: Springer.

Vallania, E. A., Garelis, P. A. y Gil, M. A. (2000). Valoración de la calidad del agua por métodos biológicos en zonas semiáridas (San Luis). Actas XVIII Congreso Nacional del Agua, Santiago del Estero: 243-244.

Venrick, E. L. (1995). Muestreo y submuestreo de fitoplancton marino y dulceacuícola. En: Alveal, K., Ferrario, M. E., Oliveira, E. C. y Sar, E. (Eds.). Manual de métodos ficológicos (pp. 200-210). Concepción, Chile: Editorial Universitaria. 